\title{
Pregnancy-associated osteoporosis with seven vertebral compression fractures, a case treated with strontium ranelate
}

\author{
Guido Zarattini ${ }^{1}$ \\ Pierangelo Buffoli ${ }^{1}$ \\ Giuliana Isabelli2 \\ Marcella Marchese ${ }^{1}$ \\ ${ }^{1}$ Orthopaedic Clinic, II Orthopaedic Division, University of \\ Brescia, Brescia, Italy \\ 2 Department of Physical Medicine and Rehabilitation, \\ "Spedali Civili" of Brescia, Brescia, Italy
}

Address for correspondence:

Guido Zarattini, MD

Orthopaedic Clinic, University of Brescia

II Orthopaedic Division

Spedali Civili of Brescia

Piazza Spedali Civili 1

25123 Brescia, Italy

Phone: +39 0303995401 ; fax: +39 030397365

E-mail: gzarattini@yahoo.it

\section{Summary}

This paper shows a case of pregnancy-associated osteoporosis (PAO) with seven vertebral compression fractures treated with strontium ranelate and supplementation of calcium and cholecalciferol. Clinical evaluation, laboratory and radiological results are analyzed in the case-report to demonstrate that strontium ranelate is a good option to restore quickly the value of bone mineral density in the treatment of a case of PAO.

KEY WORDS: pregnancy-associated osteoporosis; osteoporotic fracture; vertebral fracture; strontium ranelate.

\section{Introduction}

Pregnancy-associated osteoporosis is a rare condition first described in 1948 by Albright and Reifenstein (1) characterized by changes in calcium homeostasis and in bone metabolism that cause fragility fractures in susceptible woman (2). The incidence is estimated to be only 0.4 cases per 100.000 woman whereas the number of undiagnosed cases is probably higher (3). Its aetiology and pathogenesis have yet to be defined. Surely, pregnancy and lactation might have significant impact on bone density (4). It is therefore mandatory, during pregnant-period, consider diagnosis of pregnancy-associated osteoporosis (PAO) when hip, lumbar or thoracic spine pain are present. At the moment there are no guidelines for the therapy of PAO. All osteoporosis drugs are mainly licensed for postmenopausal patients and represent an off-label use in the treatment of premenopausal woman with PAO.

\section{Case report}

A 27-year-old primiparous and breast-feeding Caucasian woman was referred to our first-aid department 4 months after a normal vaginal delivery. She presented with acute severe back pain without history of trauma and started nearly 1 month earlier. She also had difficulties in walking that required hospital admission. The pain was localized to the back where spasm on vertebral muscles limited the motion. The severity of pain, according to the Visual Analogic Scale (VAS) was 10/10. There was no neurological deficiency. Her medical history revealed that she had menarche at 14 years of age with regular menstrual cycle. She reported that she was completely healthy before pregnancy. No osteoporosisrelated risk factors were present. However, it was noted that she did not take calcium carbonate and cholecalciferol supplementation during the pregnancy and post-partum period. Only Beta Follitropin drug was taken before pregnancy to treat infertility. On clinical examination, her height was 165 $\mathrm{cm}$ and weight was $63 \mathrm{~kg}(\mathrm{BMI}=23.1)$, with a loss of $1 \mathrm{~cm}$ comparing the value before the pregnancy. She also declared that her weight gained through the pregnancy was 14 $\mathrm{kg}$ and the birth weight of the male baby was $3850 \mathrm{~g}$. The instrumental assessment included X-rays of thoracic and lumbar spine, CT and MRI of the whole spine, bone scintigraphy and dual energy X-ray absorptiometry (DXA) for the evaluation of bone mineral density (BMD). Laboratory tests (including serum calcium, phosphate, alkaline phosphatase, thyroid and parathyroid hormone levels, liver and renal function tests, FSH, LH, estradiol, progesterone, prolactin, testosterone, osteocalcin, vitamin B12, folate, cortisol, growth hormone, ACTH, vitamin D3) were also performed and did not show any abnormal values. Visual evaluation of CT and MRI showed 7 fresh vertebral body fractures of thoracic and lumbar vertebrae $(T 1,3,4,7,9$ and L2,5) (Figure 1). Tumoral markers and bone scintigraphy excluded primary or metastatic bone diseases, as well as infectious ones. Bone densitometry measured by dual-energy X-ray absorptiometry (DXA) (Hologic Discovery Wi S/N 84400) was consistent with osteoporosis of the lumbar vertebrae (Table 1). The clinical findings, laboratory and radiological data strengthen the diagnosis of PAO. In according to diagnosis, breast-feeding was terminated and we prescribed calcium carbonate 1,000 $\mathrm{mg} /$ day and cholecalciferol $25000 \mathrm{IU} /$ week. After full and adequate explanation to the patient and her written informed consent in view of off-label-use, a therapy with strontium ranelate $2,000 \mathrm{mg} /$ day was also started. To control the pain, bed-rest and non-steroidal anti-inflammatory drugs were used for the first week then the patient used a thoracolumbosacral orthosis (TLSO) to walk. In two weeks the clinical conditions improved (VAS value $5 / 10$ ), so we decided to avoid surgery (kyphoplasty or vertebroplasty) and to continue with conservative treatment. After 3 months of treatment new X-rays was performed. These showed a consolidation of the vertebral fractures without significant spinal deformity. 


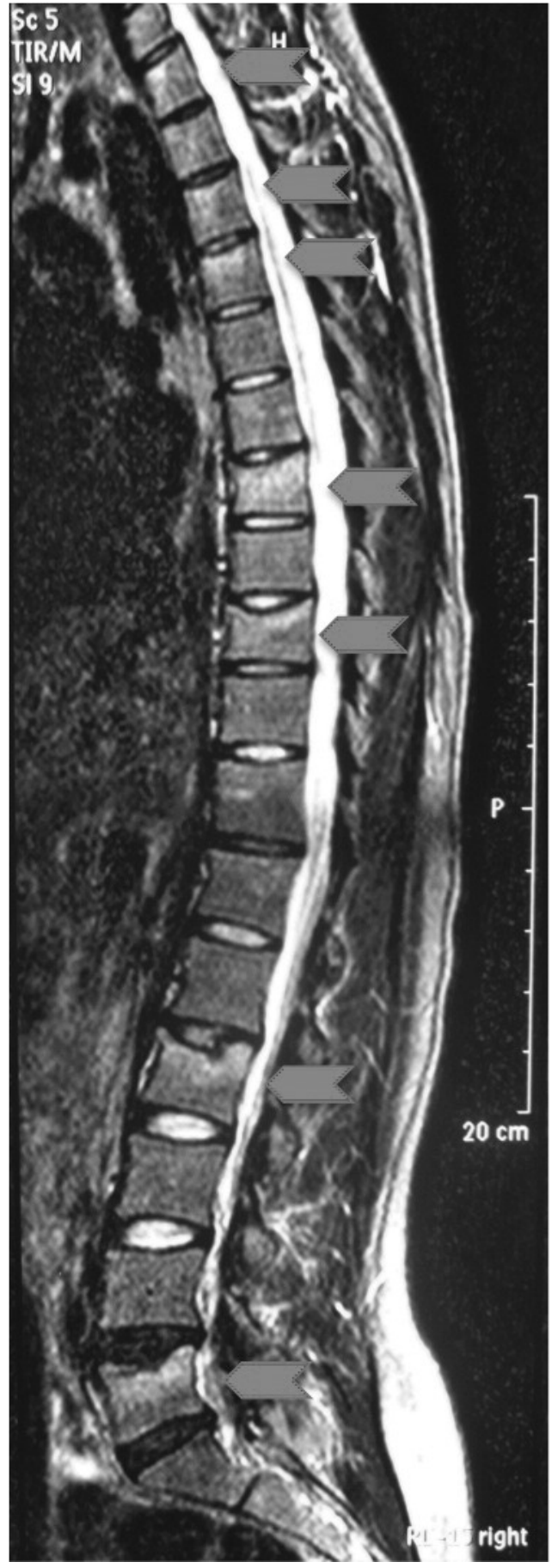

Figure $1-\mathrm{MRI}$ in lateral view of patient's spine demonstrating 7 fresh thoracic and lumbar plate fractures $(T 1,3,4,7,9$ and $L 2,5)$ grey arrows head.

The patient was almost free of back pain (VAS value 2/10), with a noticeable improvement in mobility. The TLSO was therefore gradually dismissed and she started a rehabilitation program. Six months after the onset of medical treatment, DXA measurements showed that BMD of the patient increased $17.9 \%$ at the lumbar spine and $8.6 \%$ at the proximal femur. The T-score at the total lumbar region regressed from -3.0 to -1.7 (Table 1 ). The back pain was completely absent (VAS value 0/10). After 12 months of medical treatment, another DXA measurements showed that BMD of the patient increased $31.1 \%$ at the lumbar spine and $20,2 \%$ at the proximal femur in respect to the previous control (Table 1). The patient was free of back-pain (VAS value $0 / 10$ ). A repeated $X-$ Rays showed no further vertebral fracture or progressive collapse on the previously fractured vertebrae. The therapy with strontium ranelate was therefore suspended and only calcium carbonate and cholecalciferol have been carried on. In the last follow-up at 36 months after the beginning of treatment, the DXA-values had stabilised to a constant level (Table 1). All the medical treatment was therefore stopped.

\section{Discussion}

PAO is a very rare condition that can cause fragility fractures. It usually happens during the first pregnancy, mainly in the third trimester or early post-partum period (5). The causes are not well known, but several hypotheses have been introduced. A positive family history of post-menopausal osteoporosis in PAO is seen more frequently than the controls. This could suggest that some hereditary factors are involved (5). Another hypothesis is that pregnancy is merely a stressor, which unmasks pre-existing osteoporosis (6). The change in calcium metabolism during pregnancy may contribute to the cause of PAO $(7,8)$. Under the normal environment of pregnancy and lactation, the calcium demand increases and a relative deficiency of calcium can occur (9). In our case, she did not receive calcium carbonate and cholecalciferol supplementation during pregnancy and after delivery. She had neither osteoporosis-related risk factors nor alterations in laboratory tests. Before pregnancy, our patient had a treatment of infertility with Beta Follitropin (Puregon, synthetic-FSH) for 2 weeks. FSH has been associated with BMD loss in animal models (10), but a direct effect in woman has not been demonstrated (11). The treatment with Beta Follitropin could therefore not be considered as a possible cause of PAO. We hypothesize that in our patient PAO was caused by the negative calcium balance during the last trimester and early postpartum period. Because of the lack of a definite aetiology, no standard treatment protocol has been advanced for the treatment of PAO and associated vertebral fractures. Most cases have been managed with conservative treatments: bed rest, analgesics, corset application, calcium carbonate, cholecalciferol and weaning from breast feeding $(12,13)$. Treatment of PAO with antiresorptive agents like calcitonin or bisphosphonates have been reported, with some improvement in both symptoms and BMD $(7,14,15)$. The efficacy of bisphosphonates has been demonstrated across many randomized clinical trials and/or meta-analyses in postmenopausal women with osteoporosis. However, short-term as well as long-term safety and tolerability concerns (i.e. atypical fractures and osteonecrosis of the jaw) need to be considered (16). Biphosphonates are agents which accumulate in bone for years and can cross the placenta (17), the consequences for a possible subsequent pregnancy are yet unknown. In contrast, bone anabolic therapy using parathyroid hormone (PTH 1-34, teriparatide) for the treatment of severe postmenopausal osteoporosis has been shown to significantly reduce the incidence of vertebral and non-vertebral fracture (16). It is approved for the treatment of severe postmenopausal osteoporosis for patients at high risk of fracture but limited to a 2-year course because of safety concerns with long-term use. Because of its biological nature and the short half-life, teriparatide 
Table 1 - Bone mineral density (BMD), T- and Z-score of the lumbar spine and proximal femur.

\begin{tabular}{llcccc}
\hline & & Before treatment & $\mathbf{6}$ months of treatment & 12 months of treatment & Follow-up at 36 months \\
\hline Lumbar spine & BMD & 0.624 & 0.736 & 0.965 & 1.026 \\
\hline & T-score & -3.0 & -1.7 & -0.8 & -0.7 \\
\hline Proximal femur & Z-score & -2.9 & -1.6 & -0.8 & -0.6 \\
\hline & BMD & 0.721 & 0.783 & 0.941 & -0.950 \\
\hline & T-score & -1.0 & -0.6 & -0.0 & -0.0 \\
\hline
\end{tabular}

BMD values are given as absolute values $\left(\mathrm{g} / \mathrm{cm}^{2}\right), \mathrm{T}$ - and $\mathrm{Z}$-score are in respect to average normal values.

seems to be an alternative to bisphosphonates for the treatment of PAO and it was used in some severe cases $(18,19)$. Strontium ranelate is a new treatment option for post-menopausal osteoporosis and it is considered a second-line therapy for post-menopausal women who cannot tolerate or are unable to take bisphosphonates. It has a dual effect of reducing bone resorption and increasing bone formation. As a matter of fact, it produces significant reduction in the risk of new vertebral and non-vertebral fractures, and increases both lumbar spine and femoral neck BMD (16). However, there are no available data at the moment on its effects and long-term outcome in pre-menopausal women. Only one case of PAO was treated with strontium ranelate but after a period of unsuccessful treatment with bisphosphonates (20). Balancing the advantages and the possible risks of the offlabel-use of strontium ranelate, we interdisciplinary decided for this new therapeutic option. The decision to use strontium ranelate instead of bisphosphonates was made to enable further healthy pregnancies because it is not stored in bone matrix for years like the bisphosphonates. Beside, there is no prediction of long-term follow-up adverse events concerning prenatal impairments with the use of strontium ranelate. We moreover decided not to use teriparatide for different reasons: our patient did not show a severe osteoporosis and we were worried about its already mentioned long-term safety concerns. The results that we obtained were very encouraging: remarkable increase of BMD, improvement of the clinical symptoms in few months of treatment, no occurrence of further fractures. Our case-report shows the successful use of strontium ranelate, associated with calcium and cholecalciferol, in a case of PAO with several vertebral fractures.

\section{References}

1. Albright F, Reifenstein EC Jr. The Parathyroid Glands and Metabolic Bone Disease: selected studies. Williams and Wilkins, Baltimore, 1948.

2. Hellmeyer I, Hadji P, Ziller V, Wagner U, Schmidt S. Osteoporose in der Schwangerschaft. Geburtsh Frauenheilk. 2004;64:38-45.

3. Kovacs CS. Calcium and bone metabolism during pregnancy and lactation. J Mammary Gland Biol Neoplasia. 2005;10(2):105-18.

4. Drinkwater $\mathrm{BL}$, Chesnut $\mathrm{CH}$ 3rd. Bone density changes during pregnancy and lactation in active women: a longitudinal study. Bone Miner. 1991;1 4(2):153-60.
5. Dunne F, Walters B, Marshall T, Heath DA. Pregnancy associated osteoporosis. Clin Endocrinol (Oxf). 1993;39(4):487-90.

6. Khastgir G, Studd JW, King H, Abdalla H, Jones J, Carter G, AlaghbandZadeh J. Changes in bone density and biochemical markers of bone turnover in pregnancy-associated osteoporosis. Br J Obstet Gynaecol. 1996;103(7):716-8.

7. Di Gregorio S, Danilowicz K, Rubin Z, Mautalen C. Osteoporosis with vertebral fractures associated with pregnancy and lactation. Nutrition. 2000;16(11-12):1052-5.

8. Kaur M, Pearson D, Godber I, Lawson N, Baker P, Hosking D. Longitudinal changes in bone mineral density during normal pregnancy. Bone. 2003;32(4):449-54.

9. Yoon BK, Lee JW, Choi DS, Roh CR, Lee JH. Changes in biochemical bone markers during pregnancy and puerperium. J Korean Med Sci. 2000;15(2):189-93.

10. Sun L, Peng Y, Sharrow A, lqbal J, Zhang Z, Papachristou DJ, Zaidi S, Zhu LL, Yaroslavskiy BB, Zhou H, Zallone A, Sairam MR, Kumar TR, Bo W, Braun J, Cardoso-Landa L, Schaffler MB, Moonga BS, Blair HC, Zaidi M. FSH directly regulates bone mass. 2006;125:247-60.

11. Gourlay ML, Specker BL, Li C, Hammett-Stabler CA, Renner JB, Rubin $\mathrm{JE}$, et al. Follicle-stimulating hormone is independently associated with lean mass but not BMD in younger postmenopausal women. Bone. 2012;50(1):311-6. Epub 2011 Nov 7.

12. Khovidhunkit W, Epstein S. Osteoporosis in pregnancy. Osteoporos Int. 1996;6(5):345-54.

13. Phillips AJ, Ostlere SJ, Smith R. Pregnancy-associated osteoporosis: does the skeleton recover? Osteoporos Int. 2000;11(5):449-54.

14. Hellmeyer L, Kühnert M, Ziller V, Schmidt S, Hadji P. The use of i.v. bisphosphonate in pregnancy-associated osteoporosis-case study. Exp Clin Endocrinol Diabetes. 2007;115(2):139-42.

15. O'Sullivan SM, Grey AB, Singh R, Reid IR. Bisphosphonates in pregnancy and lactation-associated osteoporosis. Osteoporos Int. 2006;17(7):1008-12.

16. Silverman S, Christiansen C. Individualizing osteoporosis therapy. Osteoporos Int. 2012;23:797-809.

17. Ornoy A, Wajnberg R, Diav-Citrin O. The outcome of pregnancy following pre- pregnancy or early pregnancy alendronate treatment. Reprod Toxicol. 2006;22(4):578-9.

18. Hellmeyer L, Boekhoff J, Hadji P. Treatment with teriparatide in a patient with pregnancy-associated osteoporosis. Gynecol Endocrinol. 2010 Oct;26(10):725-8.

19. Stumpf UC, Kurth AA, Windolf J, Fassbender WJ. Pregnancy-associated osteoporosis: an underestimated and underdiagnosed severe disease. A review of two cases in short- and long-term follow-up. Adv Med Sci. 2007;52:94-7.

20. Tanriover MD, Oz SG, Sozen T, Kilicarslan A, Guven GS. Pregnancyand lactation-associated osteoporosis with severe vertebral deformities: can strontium ranelate be a new alternative for the treatment? Spine J. 2009;9(4):e20-4. 